Research Paper

\title{
Albumin-to-Alkaline Phosphatase Ratio: A Novel Prognostic Index of Overall Survival in Cisplatin-based Chemotherapy-treated Patients with Metastatic Nasopharyngeal Carcinoma
}

Man Nie ${ }^{1,2,3^{*}}$, Peng Sun ${ }^{1,2,3^{*}}$, Cui Chen ${ }^{4}$, Xiwen Bi ${ }^{1,2,3}$, Yu Wang ${ }^{1,2,3}$, Hang Yang ${ }^{1,2,3}$, Panpan Liu ${ }^{1,2,3}$, Zhiming $\mathrm{Li}^{1,2,3}$, Yi Xia ${ }^{1,2,3 凶}$, Wenqi Jiang ${ }^{1,2,3 凶}$

1. Department of Medical Oncology, Sun Yat-sen University Cancer Center, Guangzhou 510060, Guangdong, People's Republic of China;

2. State Key Laboratory of Oncology in South China, Guangzhou 510060, Guangdong, People's Republic of China;

3. Collaborative Innovation Center for Cancer Medicine, Guangzhou 510060, Guangdong, People's Republic of China;

4. Department of Oncology, The First Affiliated Hospital, Sun Yat-Sen University, Guangzhou 510060, Guangdong, People's Republic of China

* Man Nie and Peng Sun contributed equally to this work.

$\triangle$ Corresponding authors: Yi Xia, Department of Medical Oncology, Sun Yat-sen University Cancer Center, 651 Dong Feng East Road, Guangzhou 510060, China. Tel: +86-20-87343765, Fax: +86-20-87343392. E-mail: xiayisysucc@sina.com; Wen-qi Jiang, Department of Medical Oncology, Sun Yat-sen University Cancer Center, 651 Dong Feng East Road, Guangzhou 510060, China. Tel: +86-20-87343765, Fax: +86-20-87343392. E-mail: Jiangwq2015@126.com.

(C) Ivyspring International Publisher. This is an open access article distributed under the terms of the Creative Commons Attribution (CC BY-NC) license (https://creativecommons.org/licenses/by-nc/4.0/). See http://ivyspring.com/terms for full terms and conditions.

Received: 2016.09.11; Accepted: 2016.10.29; Published: 2017.02.25

\begin{abstract}
The Albumin-to-Alkaline Phosphatase Ratio (AAPR) has been recently revealed as a prognostic index for hepatocellular carcinoma, whereas its role in metastatic nasopharyngeal cancer (NPC) remains unclear. The aim of this study was to evaluate the clinical value of AAPR in patients with metastatic NPC. We retrospectively reviewed 209 metastatic NPC patients treated with cisplatin-based regimens. Survival data were calculated using the Kaplan-Meier method and were compared using the log-rank test. Univariate and multivariate survival analyses were conducted using the Cox proportional hazards regression methodology. The optimal cutoff level of AAPR for assessing overall survival (OS) was 0.447 , which was determined by $R$ software. An AAPR less than 0.447 was significantly associated with a higher lactate dehydrogenase (LDH) level $(273$ vs. 185 $\mathrm{U} / \mathrm{L}, \mathrm{P}=0.004)$, a higher EBV DNA viral load $\left(5.59 \times 10^{5} \mathrm{vs.} 3.49 \times 10^{4} \mathrm{copies} / \mathrm{ml}, \mathrm{P}=0.001\right)$, and more liver and bone metastases $(P=0.005$ and $P=0.001$, respectively). Additionally, patients with an AAPR $<0.447$ had a shorter overall survival and progression-free survival (hazard ratio: 3.269, 95\% confidence interval: 1.710-6.248; HR: 2.295, 95\% confidence interval: 1.217-4.331, respectively) than those with an AAPR $\geq 0.447$. Our study suggested that the AAPR might be a novel prognostic factor in metastatic NPC patients treated with cisplatin-based regimens. However, a prospective study to validate its prognostic value is needed, and the mechanisms underlying the low AAPR and poor survival in metastatic NPC need to be further investigated.
\end{abstract}

Key words: Albumin-to-alkaline phosphatase ratio, cisplatin-based regimens, metastatic nasopharyngeal carcinoma.

\section{Background}

Nasopharyngeal carcinoma (NPC) is a highly malignant neoplasm with a very unique pattern of geographical distribution, with nearly $70 \%$ of new cases occurring in east and southeast Asia. ${ }^{1}$ Recently, with the advance of radiotherapy and chemotherapy, this disease has been considered a curable disease, with a 5-year overall survival (OS) exceeding $75 \%$. However, more than $20 \%$ of patients will ultimately 
develop distant metastasis after definite chemoradiotherapy. Meanwhile, 5-6\% of NPC patients are identified as having disseminated disease at initial diagnosis. ${ }^{2}$ Under this condition, systematic therapy remains the primary option. A number of treatment options for metastatic NPC have shown great success, especially cisplatin-based regimens, which have been used as first-line chemotherapies with an overall response rate of $50-80 \%{ }^{3}$ However, a significant heterogeneity of treatment outcomes has been observed; thus, the discovery of biological markers, which can conveniently predict survival to assist with clinical decision-making, is still an important topic in metastatic NPC.

Several staging systems and various serum markers have been investigated to provide prognostic information, including the TNM staging system, Epstein-Barr virus (EBV) DNA viral load, C-reactive protein, lactate dehydrogenase (LDH), albumin (ALB) and alkaline phosphatase (ALP). ${ }^{4-9}$ Some combined indexes of clinical characteristics and laboratory biomarkers have also been proven to be prognostic factors, such as the C-reactive protein-to-albumin ratio, neutrophil-to-lymphocyte ratio (NLR) and monocyte-to-lymphocyte ratio (MLR). ${ }^{10-11}$ However, the albumin-to-alkaline phosphatase ratio (AAPR), which is a novel prognostic factor for hepatocellular carcinoma (HCC), has not yet been studied in metastatic NPC patients. ${ }^{12}$ Therefore, it is of interest to determine whether AAPR can also be applied to metastatic NPC.

Thus, we conducted a retrospective cohort study aimed at examining the role of AAPR in the prognosis for metastatic NPC patients treated with cisplatin-based therapy. Additionally, we also investigated the relationship between AAPR and other clinical characteristics.

\section{Methods}

\section{Patient selection and evaluation}

We reviewed patients with metastatic NPC in Sun Yat-sen University Cancer Center from 2008 to 2011. The inclusion criteria were as follows: (1) pathologically confirmed NPC at our cancer center; (2) clinically diagnosed metastasis; (3) complete baseline comprehensive metabolic panel data, especially albumin and alkaline levels; (4) cisplatin-based chemotherapy regimens as first-line treatment; (5) Karnofsky Performance Score (KPS) greater than 60 ; (6) normal hepatic and renal function; (7) complete follow-up data; (8) available informed consent for the collection of medical information. The exclusion criteria were as follows: (1) clinically diagnosed brain metastases; (2) concurrent malignancies. A total of 209 patients with metastatic NPC between 2008 and 2011 were included in this analysis, and all of them were initially treated with cisplatin-based regimens. All of the procedures conducted in this study were in accordance with the ethical standards of the institutional research committee and with the 1964 Helsinki Declaration and its amendments. Additionally, the study protocol was approved by the ethics committee of Sun Yat-sen University Cancer Center.

Baseline clinical and laboratory assessments, including complete blood count, comprehensive metabolic panel with LDH, ALB and ALP, EBV-DNA viral load, and dedicated MRI of the nasopharynx and CT scans of the chest or PET-CT, were retrieved and reviewed from the hospital database. The AAPR was calculated by dividing the serum albumin level by the serum alkaline level. Clinical staging of the tumors was performed according to the AJCC TNM staging system (7th edition, 2010).

\section{Treatment and response evaluation}

There were three first-line chemotherapy regimens recommended in our department: 1 . TP regimen: paclitaxel $\left[175 \mathrm{mg} / \mathrm{m}^{2} \mathrm{IV}\right.$ on Day 1 of a 21-day cycle] plus cisplatin $\left(25 \mathrm{mg} / \mathrm{m}^{2}\right.$ IV on Days $1-3$ of a 21-day cycle). 2. PF regimen: cisplatin $\left(25 \mathrm{mg} / \mathrm{m}^{2}\right.$ IV on Days 1-3 of a 21-day cycle) plus 5-fluorouracil (500 mg/m² IV on Days 1-5 of a 21-day cycle). 3. TPF regimen: paclitaxel $\left(135 \mathrm{mg} / \mathrm{m}^{2}\right.$ IV on Day 1 of a 21-day cycle) plus cisplatin ( $25 \mathrm{mg} / \mathrm{m}^{2} \mathrm{IV}$ on Days $1-3$ of a 21-day cycle) plus 5-fluorouracil $\left(800 \mathrm{mg} / \mathrm{m}^{2}\right.$, continuous IV infusion for 24 hours, on Days 1-5 of a 21-day cycle). The patients were treated with one of these regimens depending on their physical status and discretion of the treating physician. The treatment response was evaluated after every two cycles of chemotherapy according to the Response Evaluation Criteria in Solid Tumors (RECIST 1.0).

\section{Statistical analysis}

Overall survival (OS) was measured from the initial diagnosis to death due to any cause or the most recent follow-up. Progression-free survival (PFS) was measured from the initial diagnosis to disease progression or death from any cause. Our final follow-up was on January 31, 2016. The optimal cutoff level of AAPR was determined by a web-based system, R software, designed by Budczied et al. ${ }^{13}$ Baseline characteristics were compared using Chi-squared test or Fisher's exact test. Survival data were calculated using the Kaplan-Meier method and were compared using the log-rank test. Univariate and multivariate survival analyses were conducted using the Cox proportional hazards regression 
methodology. Hazard ratios (HRs) with 95\% confidence intervals (CIs) and two-sided $\mathrm{P}$ values were reported. A P value $<0.05$ was considered to be statistically significant. All of the statistical analyses were performed using the Statistical Package for the Social Sciences version 19.0 (IBM, Armonk, NY, USA).

\section{Results}

\section{Patient characteristics}

Clinical characteristics at the initial diagnosis are presented in Table 1. Overall, 209 patients were included in our study, with a male predominance (male/female: 179/30). The median age of the cohort at presentation was 45 years (range: $14-72$ years). Most of the patients presented with a good physical status (KPS $\geq 90 \%$ in $81.3 \%$ of the patients). Approximately $50.2 \%$ of patients developed more than one metastasis site. Liver metastasis, lung metastasis and bone metastasis was found in 73 patients $(34.9 \%), 95$ patients $(45.5 \%)$ and 87 patients $(41.6 \%)$, respectively. The median baseline EBV DNA viral load was $4.67 \times$ $10^{4}$ copies $/ \mathrm{ml}$ (range: 0-9.19 $\times 10^{7}$ copies $/ \mathrm{ml}$ ). Additionally, the median serum ALB and ALP were $42.5 \mathrm{~g} / \mathrm{L}$ (range: $27.4-71.3 \mathrm{~g} / \mathrm{L}$ ) and 77.0 U/L (range: 9.0-644.2 U/L), respectively.

More than half of the patients (108, 51.7\%) underwent the TPF regimen as their first-line treatment, whereas 77 (36.8\%) received the PF regimen, and $24(11.5 \%)$ received the $\mathrm{TP}$ regimen. the overall response rate (complete remission and partial remission) was $70.3 \%$.

Using Cutoff Finder, a biostatistical tool, we determined 0.447 as the optimal cutoff level of the AAPR for assessing survival. Thus, the cohort was divided into two groups according to the AAPR
(AAPR $\geq 0.447, \mathrm{n}=142 ;$ AAPR < 0.447, $\mathrm{n}=67$ ). The clinicopathological characteristics of these two groups are also listed in Table 1. The clinical characteristics (including gender, age, KPS, involved sites and lung metastasis) and chemotherapy regimens were comparable between patients with an AAPR $\geq 0.447$ and patients with an AAPR $<0.447$. However, an AAPR $<0.447$ was significantly associated with a higher LDH level (273 versus $185 \mathrm{U} / \mathrm{L}, \mathrm{P}=0.004$ ), a higher EBV DNA viral load $\left(5.59 \times 10^{5}\right.$ versus $3.49 \times$ $10^{4}$ copies $\left./ \mathrm{ml}, \mathrm{P}=0.001\right)$, and more liver and bone metastases $(49.3 \%$ versus $28.2 \%, P=0.005 ; 58.2 \%$ versus $33.8 \%, \mathrm{P}=0.001$, respectively). Although the overall response rate was lower in the AAPR $<0.447$ group, no statistical significance was observed between these two groups $(64.2 \%$ vs. $73.2 \%, \mathrm{P}=$ 0.197).

\section{Survival analyses}

The median follow-up period for the entire group of patients was 16.6 months (range: 1-66.6 months). At the time of analysis, 123 patients died of NPC. The median OS (95\% CIs) and PFS (95\% CIs) in the AAPR $\geq 0.447$ group were 24.3 months (21.6-27.0) and 8.4 months (7.6-9.1), respectively. In the AAPR < 0.447 group, the median OS (95\% CI) and PFS (95\% CI) were 17.3 months (14.6-20.0) and 5.9 months (4.4-7.4), respectively. (Figure 1)

Based on a univariate analysis, the AAPR, serum LDH, EBV DNA viral load, treatment response, albumin levels and ALP were significant predictors of both OS and PFS (Tables 2 and 3). An AAPR less than 0.447 was associated with significantly inferior survival (OS, HR: 2.870, 95\% CI: 1.974-4.173, P < 0.001; PFS, HR: 1.756, 95\% CI: 1.273-2.421, $\mathrm{P}=0.001)$.

Table 1. Demographic and baseline characteristics of 209 metastatic nasopharyngeal carcinoma patients.

\begin{tabular}{|c|c|c|c|c|}
\hline Characteristic & Number (\%) & AAPR $<0.447$, Number $(\%)$ & AAPR $\geq 0.447$, Number (\%) & $P$ value \\
\hline Gender (male/female) & $179 / 30(85.6 / 14.4)$ & $60 / 7(89.6 / 10.4)$ & $119 / 23(83.8 / 16.2)$ & 0.299 \\
\hline Age, years (median/range) & $45(14-72)$ & $43(14-69)$ & $46(26-72)$ & 0.266 \\
\hline KPS $(\geq 90 /<90)$ & $170 / 39(81.3 / 18.7)$ & $50 / 17(74.6 / 25.4)$ & $120 / 22(84.5 / 15.5)$ & 0.091 \\
\hline Number of involved sites (one/multiple) & $104 / 105(49.8 / 50.2)$ & $31 / 36(46.3 / 53.7)$ & $73 / 69(51.4 / 48.6)$ & 0.554 \\
\hline Synchronous metastasis (yes/no) & $52 / 157(24.9 / 75.1)$ & $19 / 48(28.4 / 71.6)$ & $33 / 109(23.2 / 76.8)$ & 0.493 \\
\hline Liver metastasis (yes/no) & $73 / 136(34.9 / 65.1)$ & $33 / 34(49.3 / 50 / 7)$ & $40 / 102(28.2 / 71.8)$ & 0.005 \\
\hline Lung metastasis (yes/no) & $95 / 114(45.5 / 54.5)$ & $28 / 39(41.8 / 58.2)$ & $67 / 75(47.2 / 52.8)$ & 0.552 \\
\hline Bone metastasis (yes/no) & $87 / 122(41.6 / 58.4)$ & $39 / 28(58.2 / 41.8)$ & $48 / 94(33.8 / 66.2)$ & 0.001 \\
\hline Serum LDH,U/L (median/range) & $202(25-3765)$ & $273(25-3765)$ & 185 (111-1909) & 0.004 \\
\hline Albumin (median/range) & $42.5(27.4-71.3)$ & $40(27.4-49.0)$ & $43.8(30.8-71.3)$ & $<0.001$ \\
\hline ALP (median/range) & $77(9.0-644.2)$ & $121(77.0-644.2)$ & $65.6(9.0-102.6)$ & $<0.001$ \\
\hline EBV-DNA, copies/ml (median/range) & $4.67 \times 104 / 0-9.19 \times 107$ & $5.59 \times 105 / 0-9.19 \times 107$ & $3.49 \times 104 / 0-8.87 \times 106$ & 0.001 \\
\hline Chemotherapy regimen (TPF/PF/TP) & $108 / 77 / 24(51.7 / 36.8 / 11.5)$ & $30 / 29 / 8(44.8 / 44.3 / 11.9)$ & $78 / 48 / 16(54.9 / 33.8 / 11.3)$ & 0.361 \\
\hline Treatment response $(\mathrm{CR}+\mathrm{PR} / \mathrm{PD}+\mathrm{SD})$ & $147 / 62(70.3 / 29.7)$ & $43 / 24(64.2 / 35.8)$ & $104 / 38(73.2 / 26.8)$ & 0.197 \\
\hline
\end{tabular}


Table 2. Univariate and multivariate analyses of the prognostic factors of overall survival for metastatic nasopharyngeal carcinoma patients.

\begin{tabular}{|c|c|c|c|c|c|}
\hline \multirow[t]{2}{*}{ Variable } & \multicolumn{2}{|c|}{ Univariate } & \multicolumn{2}{|c|}{ Multivariate } & \multirow{2}{*}{$\begin{array}{l}\text { Bootstrap } \\
\text { p value }\end{array}$} \\
\hline & $p$ value & $\mathrm{HR}(95 \% \mathrm{CI})$ & $\mathrm{p}$ value & $\mathrm{HR}(95 \% \mathrm{CI})$ & \\
\hline Gender (male/female) & 0.652 & $1.138(0.650-1.993)$ & & & \\
\hline Age, years $(<50 / \geq 50)$ & 0.779 & $1.057(0.718-1.555)$ & & & \\
\hline KPS $(\geq 90 /<90)$ & 0.636 & $0.891(0.551-1.440)$ & & & \\
\hline Number of involved sites (one/multiple) & 0.165 & $1.288(0.901-1.840)$ & & & \\
\hline Synchronous metastasis (yes/no) & 0.529 & $0.872(0.570-1.335)$ & & & \\
\hline Liver metastasis (yes/no) & 0.956 & $0.990(0.684-1.432)$ & & & \\
\hline Lung metastasis (yes/no) & 0.862 & $1.032(0.723-1.474)$ & & & \\
\hline Bone metastasis (yes/no) & 0.083 & $1.375(0.959-1.970)$ & & & \\
\hline Smoking (yes/no) & 0.959 & $1.009(0.707-1.440)$ & & & \\
\hline $\operatorname{AAPR}(<0.447 / \geq 0.447)$ & $<0.001$ & $2.870(1.974-4.173)$ & $<0.001$ & $3.269(1.710-6.248)$ & 0.001 \\
\hline Serum LDH,U/L (<212/ $\geq 212)$ & 0.002 & $1.756(1.226-2.514)$ & 0.400 & $1.180(0.802-1.737)$ & 0.430 \\
\hline EBV-DNA , copies/ml $(<4.9 \times 104 / \geq 4.9 \times 104)$ & $<0.001$ & $3.660(2.516-5.324)$ & $<0.001$ & $2.993(2.014-4.449)$ & 0.001 \\
\hline Chemotherapy regimen (PF/TP/TPF) & 0.446 & $0.910(0.706-1.173)$ & & & \\
\hline Treatment response $(\mathrm{CR}+\mathrm{PR} / \mathrm{PD}+\mathrm{SD})$ & $<0.001$ & $0.469(0.323-681)$ & 0.037 & $0.664(0.451-0.976)$ & 0.056 \\
\hline Albumin, g/L $(<40 / \geq 40)$ & 0.002 & $1.824(1.248-2.664)$ & 0.633 & $1.107(0.729-1.681)$ & 0.677 \\
\hline ALP, U/L (<104/ $\geq 104)$ & $<0.001$ & $2.270(1.541-3.345)$ & 0.139 & $0.614(0.322-1.172)$ & 0.091 \\
\hline
\end{tabular}

Table 3. Univariate and multivariate analyses of prognostic factors of progression-free survival for metastatic nasopharyngeal carcinoma patients.

\begin{tabular}{|c|c|c|c|c|c|}
\hline \multirow[t]{2}{*}{ Variable } & \multirow{2}{*}{$\begin{array}{l}\text { Univariate } \\
\mathrm{p} \text { value }\end{array}$} & & \multicolumn{2}{|c|}{ Multivariate } & \multirow{2}{*}{$\begin{array}{l}\text { Bootstrap } \\
\text { p value }\end{array}$} \\
\hline & & $\mathrm{HR}(95 \% \mathrm{CI})$ & $\mathrm{p}$ value & $\mathrm{HR}(95 \% \mathrm{CI})$ & \\
\hline Gender (male/female) & 0.505 & $1.075(0.869-1.329)$ & & & \\
\hline Age, years $(<50 / \geq 50)$ & 0.722 & $1.063(0.761-1.458)$ & & & \\
\hline KPS $(\geq 90 /<90)$ & 0.317 & $1.218(0.828-1.792)$ & & & \\
\hline Number of involved sites (one/multiple) & 0.055 & $1.355(0.993-1.848)$ & & & \\
\hline Synchronous metastasis (yes/no) & 0.831 & $1.039(0.733-1.472)$ & & & \\
\hline Liver metastasis (yes/no) & 0.507 & $0.900(0.659-1.229)$ & & & \\
\hline Lung metastasis (yes/no) & 0.146 & $1.248(0.926-1.682)$ & & & \\
\hline Bone metastasis (yes/no) & 0.273 & $1.183(0.876-1.599)$ & & & \\
\hline Smoking (yes/no) & 0.748 & $1.050(0.780-1.415)$ & & & \\
\hline $\operatorname{AAPR}(<0.447 / \geq 0.447)$ & 0.001 & $1.756(1.273-2.421)$ & 0.001 & $2.295(1.217-4.331)$ & 0.042 \\
\hline Serum LDH,U/L (<212/ $\geq 212)$ & 0.019 & $1.436(1.061-1.943)$ & 0.463 & $1.142(0.801-1.627)$ & 0.484 \\
\hline EBV-DNA , copies/ml $(<4.9 \times 104 / \geq 4.9 \times 104)$ & $<0.001$ & $2.725(1.972-3.766)$ & $<0.001$ & $2.018(1.421-2.864)$ & 0.001 \\
\hline Chemotherapy regimen (PF/TP/TPF) & 0.599 & $1.060(0.854-1.315)$ & & & \\
\hline Treatment response (CR+PR/PD+SD) & $<0.001$ & $0.245(0.171-0.352)$ & $<0.001$ & $0.292(0.200-0.425)$ & 0.001 \\
\hline Albumin, g/L $(<40 / \geq 40)$ & 0.007 & $1.560(1.127-2.160)$ & 0.774 & $1.056(0.727-1.534)$ & 0.805 \\
\hline ALP, U/L (<104/ $\geq 104)$ & 0.025 & $1.486(1.050-2.105)$ & 0.070 & $0.557(0.296-1.048)$ & 0.157 \\
\hline
\end{tabular}
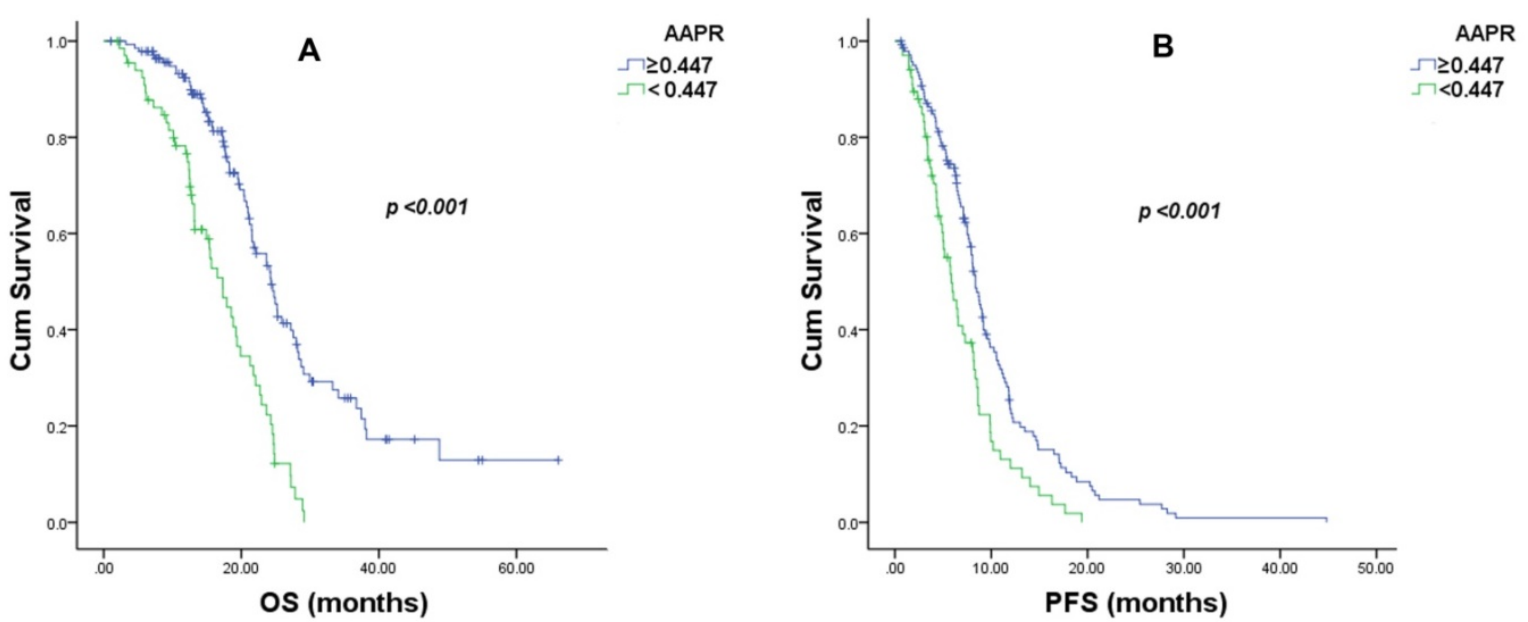

Figure 1. Overall survival (OS) and progression-free survival (PFS) curves of 209 metastatic nasopharyngeal carcinoma patients with different pretreatment values of the albumin-to-alkaline phosphatase ratio (AAPR). (A). The median OS was $24.3 \pm 1.4$ months ( $95 \%$ confidence interval: $21.6-27.0$ ) in the AAPR $\geq 0.447$ group and $17.3 \pm 1.4$ months ( $95 \% \mathrm{Cl}: 14.6-20.0)$ in the AAPR < 0.447 group $(X=33.287, P<0.001)$. (B) The median PFS was $8.4 \pm 0.4$ months $(95 \%$ confidence interval: $7.6-9.1)$ in the AAPR $\geq 0.447$ group and $5.9 \pm 0.8$ months $(95 \% \mathrm{Cl}: 4.4-7.4)$ in the AAPR $<0.447$ group $(X=12.139, P<0.001)$. 
Neither the metastasis sites nor the chemotherapy regimens displayed a significant prognostic impact. In the multivariate analysis, an AAPR less than 0.447 was found to be an independent predictor of both OS and PFS (OS, HR: 3.269, 95\%; CI: 1.710-6.248, $\mathrm{P}<0.001$; PFS, HR: 2.295, 95\% CIs: 1.217-4.331, $\mathrm{P}=0.001)$. Other independent prognostic factors included the EBV DNA viral load and treatment response for both OS and PFS. As ALB and ALP were independent prognostic indexes in metastatic NPC patients, we compared the areas under the receiver operating characteristic curve (ROCs) for AAPR, ALB and ALP. The AAPR showed a relatively higher AUC value (0.634) than those of ALP (0.604) and ALB (0.559) (Figure 2).

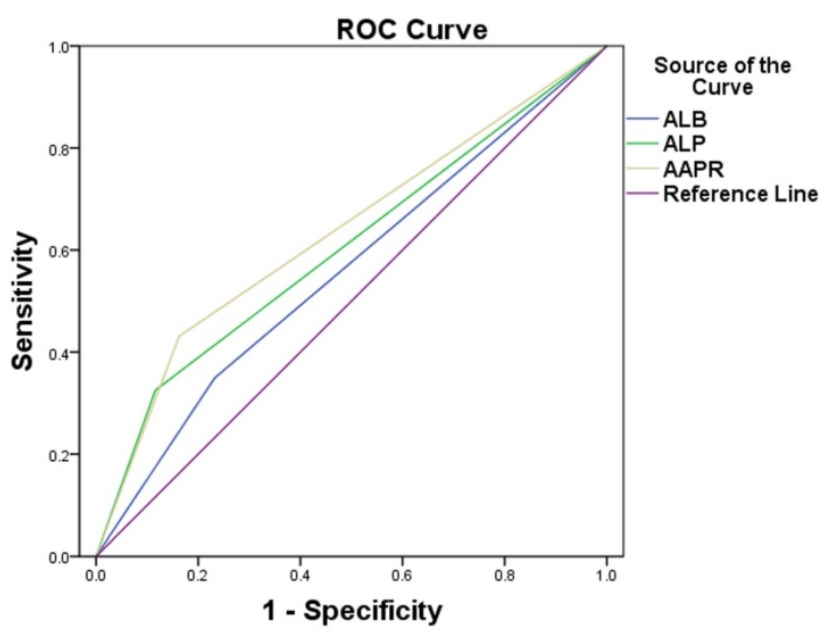

Figure 2. Comparisons of the area under the receiver operating curve for survival status among albumin-to-alkaline phosphatase ratio (AAPR), albumin level and alkaline phosphatase level. The areas were 0.634, 0.559 and 0.604, respectively.

\section{Discussion}

In this study, we focused exclusively on the prognostic value of the AAPR in cisplatin-based therapy-treated patients with metastatic NPC. To our knowledge, this is the first study to analyze the correlation between the AAPR and metastatic NPC. Our findings suggest that the AAPR is an independent prognostic indicator for patients with metastatic NPC; patients with an AAPR less than 0.447 exhibited inferior OS and PFS.

ALB, the most abundant serum protein, is considered a nutritional index with the ability to stabilize cell growth and DNA replication, buffer various biochemical changes, and exert antioxidant effects against carcinogens. ${ }^{14}$ In addition, malnutrition, which is reflected by low ALB, can weaken human defense mechanisms such as cellular and humoral immunity and phagocytic function, leading to the increasing possibility of infection and the poor response to anti-cancer treatment. ${ }^{15}$ Recently, ALB has become a popular biomarker for the prediction of survival in various malignancies, including colorectal cancer, osteosarcoma, hepatocellular carcinoma, renal cell carcinoma and prostate cancer. ${ }^{16-20} \mathrm{Li}$ et al. reported that NPC patients with a pretreatment ALB level less than 43 $\mathrm{g} / \mathrm{L}$ had a poor prognosis. The underlying mechanisms included malnutrition and an imbalanced tumor microenvironment. A high concentration of interleukin 6 (IL-6) and tumor necrosis factor (TNF) can modulate the production of albumin in hepatocytes and increase the permeability of the microvasculature. ${ }^{21}$ Furthermore, EBV, a well-known prognostic factor in NPC regardless of stage, has the ability to stimulate the release of pro-inflammatory cytokines, including IL-6 and TNF, which pathologically intensifies these processes. ${ }^{22}$

Alkaline phosphatase (ALP) is a hydrolase enzyme that can dephosphorylate various types of molecules, including nucleotides, proteins and alkaloids. ${ }^{23}$ Although almost all tissues throughout the entire body contain this enzyme, it is specifically concentrated in the bone, liver, bile duct, kidney and placenta. ${ }^{24}$ More ALP will be released into the blood stream during some pathological conditions, including pregnancy, hepatocellular carcinoma, kidney disease and bone metastasis. ${ }^{25-28}$ Particularly, elevated ALP is frequently reported in bone metastasis in certain malignancies, including osteosarcoma, breast cancer and prostate cancer. ${ }^{20,29-30}$ Jin et al reported that serum ALP predicted a worse survival outcome in patients with skeletal and/or liver metastasis of NPC. ${ }^{31}$

Anthony and colleagues first proposed the concept of the AAPR and proved that the AAPR was an independent prognostic factor for overall survival and disease-free survival for patients with hepatocellular carcinoma receiving curative surgery. ${ }^{12}$ Additionally, we hypothesized that merging ALB and ALP into a new index may have a novel prognostic value and better predicted the survival of patients with metastatic NPC. In our study, a 0.447 cut-off value for AAPR was adopted to predict the survival in metastatic NPC patients. Both univariate analysis and multivariate analysis showed that an AAPR less than 0.447 is associated with poor prognosis and a high EBV DNA viral load. A low AAPR may reflect the patient's malnutrition status, impressed immunity, and relatively severe disease condition such as liver or bone metastasis; a high EBV DNA viral load may lead to imbalanced immunity in the tumor microenvironment as previously reported, which may promote tumor growth, invasion and 
metastasis. The AAPR is a combination of these predictors of adverse outcomes, which may enhance its prognostic value; besides, we found the AAPR had a higher AUC value than those of ALP and ALB. Thus, it is reasonable that the AAPR is a superior predictor factor of survival compared to other factors of nutrition or inflammation, like ALB and ALP. Considering the toxicities of intensive regimens and similar efficacy of $\mathrm{TP} / \mathrm{PF} / \mathrm{TPF}$ regimens, it is recommended that TP or PF may be a better choice for low-AAPR-value patients.

Generally speaking, there were several advantages in our study. First, we investigated the prognostic value of AAPR in metastatic NPC and its correlation with other clinical factors, which had not been reported in previous publications. Second, all of the enrolled patients were treated with cisplatin-based regimens, which, to a large extent, minimized the potential influence from the variation of different treatment modalities. Third, the AAPR can be easily and objectively calculated from a comprehensive metabolic panel. Finally, the AAPR as a continuous index showed a better predictive value than the simple addition of albumin and ALP.

The limitations of this study should be acknowledged. First, this is a retrospective and single-center study; no independent cohorts were introduced to confirm the prognostic value of AAPR. Second, the optimal cutoff for AAPR also requires external validation. Third, whether the dynamic change in the AAPR during the treatment course can predict the prognosis remains unknown. Future prospective clinical trials and basic research can emphasize and uncover the value and mechanisms regarding the AAPR.

The current study indicates that the AAPR is a prognostic index for metastatic NPC patients treated with cisplatin-based palliative chemotherapy. In addition, a prospective study to validate its prognostic value is warranted. The mechanisms underlying the close relationship between a lower AAPR and inferior survival in metastatic NPC need to be further investigated.

\section{Abbreviations}

AAPR: Albumin-to-alkaline phosphatase ratio; ALB: albumin; ALP: alkaline phosphatase; EBV: Epstein-Barr virus; LDH: lactate dehydrogenase; HCC: hepatocellular carcinoma; KPS: Karnofsky Performance Score; MLR: monocyte-to-lymphocyte ratio; NPC: nasopharyngeal carcinoma; NLR: neutrophil-to-lymphocyte ratio; OS: overall survival; PFS: progression-free survival; ROC: receiver operating characteristic curve.

\section{Competing Interests}

The authors have declared that no competing interest exists.

\section{References}

1. Chen $\mathrm{W}$, Zheng R, Baade PD, et al. Cancer statistics in China. CA Cancer J Clin. 2015; 2016: 89-171.

2. Chiesa F, De Paoli F. Distant metastases from nasopharyngeal cancer. ORL J Otorhinolaryngol Relat Spec. 2001; 63: 214-216.

3. Bensouda Y, Kaikani W, Ahbeddou N, et al. Treatment for metastatic nasopharyngeal carcinoma. Eur Ann Orl Head Neck Dis. 2011; 128: 79-85.

4. Sun P, Chen C, Cheng YK, et al. Serologic biomarkers of Epstein-Barr virus correlate with TNM classification according to the seventh edition of the UICC/AJCC staging system for nasopharyngeal carcinoma. Eur Arch Otorhinolaryngol. 2014; 271: 2545-2554.

5. Jin Y, Cai XY, Cai YC, et al. To build a prognostic score model containing indispensible tumour markers for metastatic nasopharyngeal carcinoma in an epidemic area. Eur J Cancer. 2012; 48: 882-888.

6. Jin $\mathrm{Y}, \mathrm{Ye} \mathrm{X}$, Shao $\mathrm{L}$, et al. Serum lactic dehydrogenase strongly predicts survival in metastatic nasopharyngeal carcinoma treated with palliative chemotherapy. Eur J Cancer. 2013; 49: 1619-1626.

7. Wang WY, Twu CW, Chen $\mathrm{HH}$, et al. Plasma EBV DNA clearance rate as a novel prognostic marker for metastatic/recurrent nasopharyngeal carcinoma. Clin Cancer Res. 2010; 16: 1016-1024.

8. Xie Y, Wei ZB, Duan XW. Prognostic value of pretreatment serum alkaline phosphatase in nasopharyngeal carcinoma. Asian Pac J Cancer Prev. 2014; 15: 3547-3553.

9. Li G, Gao J, Tao YL, et al. Increased pretreatment levels of serum LDH and ALP as poor prognostic factors for nasopharyngeal carcinoma. Chin J Cancer. 2012; 31: 197-206.

10. Jin Y, Ye X, He C, et al. Pretreatment neutrophil-to-lymphocyte ratio as predictor of survival for patients with metastatic nasopharyngeal carcinoma. Head Neck. 2015; 37: 69-75.

11. Li J, Jiang R, Liu WS, et al. A large cohort study reveals the association of elevated peripheral blood lymphocyte-to-monocyte ratio with favorable prognosis in nasopharyngeal carcinoma. PLOS ONE. 2013; 8: e83069.

12. Chan AW, Chan SL, Mo FK, et al. Albumin-to-alkaline phosphatase ratio: A novel prognostic index for hepatocellular carcinoma. Dis Markers. 2015; 2015: 564057.

13. Budczies J, Klauschen F, Sinn BV, et al, Denkert C. Cutoff finder: A comprehensive and straightforward Web application enabling rapid biomarker Cutoff optimization. PLOS ONE. 2012; 7: e51862.

14. Arroyo V, García-Martinez R, Salvatella X. Human serum albumin, systemic inflammation, and cirrhosis. J Hepatol. 2014; 61: 396-407.

15. Chandra RK. Nutrition and immunology: From the clinic to cellular biology and back again. Proc Nutr Soc. 1999; 58: 681-683.

16. Nazha B, Moussaly E, Zaarour M, et al. Hypoalbuminemia in colorectal cancer prognosis: Nutritional marker or inflammatory surrogate? World J Gastrointest Surg. 2015; 7: 370-377.

17. $\mathrm{Yi} \mathrm{JH}$, Wang D, Li ZY, et al. C-reactive protein as a prognostic factor for human osteosarcoma: A meta-analysis and literature review. PLOS ONE. 2014; 9: e94632.

18. Hiraoka A, Kumada T, Nouso K, et al. Proposed New sub-grouping for intermediate-stage hepatocellular carcinoma using albumin-bilirubin grade. Oncology. 2016; [Epub ahead of print].

19. Chen Z, Shao Y, Fan M, et al. Prognostic significance of preoperative C-reactive protein: Albumin ratio in patients with clear cell renal cell carcinoma. Int J Clin Exp Pathol. 2015; 8: 14893-14900.

20. Chi KN, Kheoh T, Ryan CJ, et al. A prognostic index model for predicting overall survival in patients with metastatic castration-resistant prostate cancer treated with abiraterone acetate after docetaxel. Ann Oncol. 2016; 27: 454-460.

21. Li G, Gao J, Liu ZG, et al. Influence of pretreatment ideal body weight percentile and albumin on prognosis of nasopharyngeal carcinoma: Long-term outcomes of 512 patients from a single institution. Head Neck. 2014; 36: 660-666.

22. Ou C, Sun $\mathrm{Z}$, Zhang $\mathrm{H}$, et al. SPLUNC1 reduces the inflammatory response of nasopharyngeal carcinoma cells infected with the EB virus by inhibiting the TLR9/NF-кB pathway. Oncol Rep. 2015; 33: 2779-2788.

23. Reiss I, Inderrieden D, Kruse K. Measurement of skeletal specific alkaline phosphatase in disorders of calcium metabolism in childhood. Monatsschr Kinderheilkd. 1996; 144: 885-890.

24. Greene PJ, Sussman HH. Structural comparison of ectopic and normal placental alkaline phosphatase. Proc Natl Acad Sci U S A. 1973; 70: 2936-2940.

25. Al Mamari S, Djordjevic J, Halliday JS, Chapman RW. Improvement of serum alkaline phosphatase to $<1.5$ upper limit of normal predicts better outcome and reduced risk of cholangiocarcinoma in primary sclerosing cholangitis. J Hepatol. 2013; 58: 329-334.

26. Damera S, Raphael KL, Baird BC, et al. Serum alkaline phosphatase levels associate with elevated serum C-reactive protein in chronic kidney disease. Kidney Int. 2011; 79: 228-233. 
27. Lu Y, Lu Q, Chen HL. Diagnosis of primary liver cancer using lectin affinity chromatography of serum alkaline phosphatase. J Exp Clin Cancer Res. 1997; 16: 75-80.

28. Bashiri A, Katz O, Maor E, et al. Positive placental staining for alkaline phosphatase corresponding with extreme elevation of serum alkaline phosphatase during pregnancy. Arch Gynecol Obstet. 2007; 275: 211-214.

29. Han J, Yong B, Luo C, et al. High serum alkaline phosphatase cooperating with MMP-9 predicts metastasis and poor prognosis in patients with primary osteosarcoma in Southern China. World J Surg Oncol. 2012; 10: 37.

30. Liu X, Meng QH, Ye Y, et al. Prognostic significance of pretreatment serum levels of albumin, $\mathrm{LDH}$ and total bilirubin in patients with non-metastatic breast cancer. Carcinogenesis. 2015; 36: 243-248.

31. Jin $Y$, Yuan MQ, Chen JQ, et al. Serum alkaline phosphatase predicts survival outcomes in patients with skeletal metastatic nasopharyngeal carcinoma. Clinics (Sao Paulo). 2015; 70: 264-272. 